\title{
Optimization of Hexagonal Boron-Doped Silicate Photonic Crystal Fiber To Obtain Near Zero Flattened Dispersion For Nonlinear Waves By Finite Difference Method
}

\section{Achyutesh Dixit ( $\square$ acdixit55@gmail.com )}

Muzaffarpur Institute of Technology https://orcid.org/0000-0003-1147-2524

\section{S. Tiwari}

Vignan's Foundation for Science Technology and Research

Ajay Kumar Vyas

Adani Institute of Infrastructure Management

Rajesh Kumar

Muzaffarpur Institute of Technology

Atul Pandey

Kashi naresh govt pg college gyanpur

\section{Research Article}

Keywords: Dispersion, Doped Fiber, Finite domain method, Nonlinear, Photonic Crystal Fiber

Posted Date: December 15th, 2021

DOI: https://doi.org/10.21203/rs.3.rs-1090837/v1

License: (c) (i) This work is licensed under a Creative Commons Attribution 4.0 International License.

Read Full License 


\section{Abstract}

The $\mathrm{B}_{2} \mathrm{O}_{3}$-doped silicate photonic crystal fiber (PCF) containing small core and dielectric rods made of lead silicate SF57 has been most intensively investigated for different pump signals centered at $0.65 \mathrm{~mm}$, communication band. This type of doping has been carried out to significantly diminish the upgraded refractive index of silica which enhances special capabilities that lead to an outstanding potential to PCF for the profoundly intense field in the optical Kerr effect. In this paper, the mode analysis has been done by solving a nonlinear wave equation for a Gaussian input beam using the finite difference method under analytical boundary conditions. Numerical results reveal that very minute doping of $\mathrm{B}_{2} \mathrm{O}_{3}$ is enough in giving prolonged confinement of beam with flattened dispersion due to ultra-low change in nonlinear behaviour.

\section{Introduction}

The photonic crystal fibre (PCF), which is used to build various sensors, has a wide range of applications in the field of photonics [1-2]. The various structures of the PCF are reported [3] so far, the hexagonal structure [4] is widely used in various applications and it is the most preferable design [5]. Photonic crystal fibers (PCFs) offer great flexibility in terms of dispersion and mode profile, along with proposing prospects for the optimize other parameters [6].

The production of the PCF using single and mixed alkali oxide glasses with varied optical absorption, photonic band gaps, energy, and two-photon absorption coefficients, as well as visible and near-infrared (NIR) fluorescence properties, including visible luminescence decay, was investigated [7].

The role of doping for PCF fabrication has significantly contributed in optimizing the PCF properties [8] such as effective refractive index, dispersion, effective area, and normalized frequency.

Many researchers have investigated various doped PCF for example thulium-doped [9], erbium-doped fiber [10], ytterbium-doped [11], phosphosilicate and germanosilicate PCF [12] and many more for different applicability. The performance parameter of PCFs can be controlled by using design parameters of PCF such as the pitch of air holes, inner and outer hole diameters, as well as doping level can be modified the dispersion [13]. J. Olszewski, et. al. presented a novel dual concentric core PCF doped inclusions, to compensate the dispersion for the inner and outer core, using $\mathrm{GeO}_{2}$ and $\mathrm{B}_{2} \mathrm{O}_{3}$ respectively. The measured value of chromatic dispersion equal $-320 \mathrm{ps} / \mathrm{nm}-\mathrm{km}$ at $1.55 \mu \mathrm{m}$ [14].

Another methodology was proposed to achieve the ultra-flat zero-dispersion using porous-core PCF, with elliptical air-holes in the core, and round-corner hexagonal air-holes in cladding, for efficiently transmitting polarization-maintaining terahertz waves that showed its near-zero ultra-flattened dispersion $(-0.01 \pm 0.06$ $\mathrm{ps} / \mathrm{THz}-\mathrm{cm}$ ) and birefringence higher than $7.0 \times 10^{-2}$ at $\mathrm{THz}$ order [15].

Yani Zhang et. al proposed a porous-core PCF with six-ring hexagonal lattice circular air holes in the cladding and asymmetrical rectangular air holes in the core for realizing low-loss polarization terahertz 
transmission. The near-zero flattened dispersion of $-0.01 \pm 0.02 \mathrm{ps} / \mathrm{THz} / \mathrm{cm}$ and birefringence of $7.1 \times 10^{-2}$ is accomplished over the THz frequency range [16]. In some models, by optimizing the number of holes, radius, and pitch for the low dispersion. By altering the shape of the holes as elliptical and stellar holes and impurity is exploited in the PCF core as a defect are also reported to reduce the dispersion [17]. Double-clad $\mathrm{AsSe}_{2}$-based PCFs are also suggested for ultra-flat near-zero dispersion at the Mid-IR range. It presented nearly zero all-normal dispersion of near $\mathrm{D} \sim-3.4 \mathrm{ps}(\mathrm{nm} . \mathrm{km})^{-1}$ equivalent to minimum group velocity dispersion at $6.9 \mu \mathrm{m}[18]$.

The dispersion represents the amount of pulse broadening in practical applications and is affected by the background material and the structure of the PCF. The zero dispersion wavelengths around the $1.5 \mu \mathrm{m}$ communications window having with higher-order dispersion (around $0.25 \mathrm{ps} / \mathrm{nm} 2 \mathrm{~km}$ ). W.H. Reeves et.al. presented PCF that has flattened, near-zero, dispersion over a very much wider wavelength range from $1 \mu \mathrm{m}$ to $1.6 \mu \mathrm{m}$. [19].

The effective area is a parameter of great significance with respect to PCF. A PCF's single mode or multimode operation can be determined through the values of effective area. Mortensen, N. A., et al [20] in their work, have proposed a large mode area PCF. Study has been done on improving the mode area of the PCF by incorporating higher index rods in the cladding region while the PCF still operates in the single mode manner. A dispersion flattened PCF with a large effective area $\left(\sim 100 \mu \mathrm{m}^{2}\right)$ and low confinement loss has been studied by [21] in their work. The flattened dispersion is observed in all the telecommunication bands. The air hole structure of the cladding portion of the PCF is embedded in the core region too, with different diameters of air holes. In [22], the authors have numerically studied the properties of PCF by varying the air holes size in the cladding region. Estimation of effective area at different parameters of PCF has been done particularly the pitch of the PCF. The PCF is composed of triangular air-hole lattice but the approach is true for microstructured fibers, in common.

The normalized frequency decides the mode of operation of the PCF viz. single mode or multi-mode. By choosing the design parameters of PCF carefully, normalized frequency can be made to remain under a threshold value, making the PCF to only allow a single mode to pass through it. PCF as a low loss optical waveguide has been studied by [23] in their work. They have controlled the normalized frequency of the fiber by specific arrangement of air holes in its cladding region. The dispersion and signal loss occurring in PCF is studied with help of equations by [24] in their work. The wavelengths chosen for the analysis of the fiber are 850, 1300 and $1550 \mathrm{~nm}$. Normalized frequency parameter has also been calculated with the help of its equation.

The $\mathrm{B}_{2} \mathrm{O}_{3}$ provides thermal stability [25] and also increases the polarization bandwidth for boron-doped PCF [26]. Such doping can control the spectral width which is significant control the dispersion. Such as narrow band $0.65 \mu \mathrm{m}$ PCF will be utilized in the optical pyrometers for high-temperature measurement.

In this work, we extended our previous work [27] regarding the effect of material dispersion of square PCF. In this article, we presented the optimization of dispersion for hexagonal PCF using boron-doped silicate 


\section{Parameter Of Photonic Crystal Fiber}

The cross-sectional view of the seven layers of hexagonal PCF is shown in figure 1 . This has a single substrate of silica glass with different concentrations of $\mathrm{B}_{2} \mathrm{O}_{3}$. The core of the fiber is solid silica and cladding consists of seven rings of air holes in a hexagonal lattice with identical sizes. Four important parameters are considered to model the PCF such as effective refractive index, dispersion, effective area, and normalized frequency.

The Sellmeier equation is used to express the refractive index of pure silica and doped silica is given in Eqn. (1) [28],

$$
n^{2}(\lambda)=1+\sum_{i=1}^{3} \frac{A_{i} \lambda^{2}}{\lambda^{2}-\lambda_{i}^{2}}(1)
$$

where $A_{i}$ constant obtained experimentally and $\lambda_{i}$ the wavelength of resonances. The six Sellmeier coefficients are $A_{i}$ and where $i=1,2,3$ will characterize the refractive index $n(\lambda)$ and material dispersion of glass. The Sellmeier coefficients of pure silica and doped silica are summarized in Table 1.

Table 1

Sellmeier coefficients for the boric anhydride doped PCF [29].

\begin{tabular}{|c|c|c|c|c|c|c|}
\hline \multirow{2}{*}{$\begin{array}{l}\text { Different } \\
\text { Mol.\% } \\
\text { of } \mathrm{B}_{2} \mathrm{O}_{3}\end{array}$} & \multicolumn{6}{|c|}{ Sellmeier coefficients } \\
\hline & $\mathrm{B}_{1}$ & $\mathrm{~B}_{2}$ & $\mathrm{~B}_{3}$ & $\mathrm{C}_{1}$ & $\mathrm{C}_{2}$ & $\mathrm{C}_{3}$ \\
\hline 3.0 & 0.6935408 & 0.9111432 & 0.01578530 & 97.93387 & 0.01578530 & 97.93387 \\
\hline 3.5 & 0.6929642 & 0.4052977 & 0.9154064 & 0.003658351 & 0.01536631 & 97.93383 \\
\hline 13.0 & 0.690618 & 0.401996 & 0.00383161 & 0.01529229 & 0.01529229 & 82.79107308 \\
\hline
\end{tabular}

In a communication system, the dispersion has noteworthy issues in both the linear and the nonlinear regimes and even for ultrashort soliton pulse. The order of dispersion is also considering when dealing with very broad optical spectra. The dispersion properties of the PCF is varying with air hole dimensions but this will be responsible for the negative dispersion generation [30].

At the same time, it allows us to include explicitly the chromatic dispersion of the material, and therefore to calculate the real dispersion of the PCF as given in Eqn. (2) [30 - 32]

$$
D_{\text {mat }}=-\frac{\lambda}{c} \frac{d^{2} n_{e f f}}{d \lambda^{2}}(2)
$$

where the effective refractive index of the mode is given by the Eq. (1). 
Fundamental modes as a function of normalized frequency. It is determined by the mathematical relation as given in Eqn. (3) [32]

$$
V_{\text {eff }}=\frac{2 \Pi}{\lambda} \Lambda\left(n_{\text {core }}^{2}-n_{\text {eff }}^{2}\right)(3)
$$

Where $n_{\text {core }}$ is the refractive index of the core, $n_{\text {eff }}$ is the effective refractive index, and $\Lambda$ is the pitch.

The confinement loss and dispersion characteristics of PCFs also be depending on the effective area. The confinement loss tends to increase in a conventional dispersion-flattened PCF (DF-PCF) that has uniform air holes [33]. The is an effective area (Aeff) is given by the mathematical relation as given in Eqn. (4) [33, 34],

$$
A_{e f f}=\frac{\left(\iint E^{2} d x d y\right)^{2}}{\iint|E|^{4} d x d y}(4)
$$

\section{Results \& Discussion}

The effective refractive index $\left(\mathrm{n}_{\text {eff }}\right)$ in a periodic structure with several dielectrics rods can be measured using the finite difference technique [FDM]. The air-dielectric rods in the proposed PCF have been substituted with high-index lead silicate SF57 material for strong PBG guidance which is suitable for a highly nonlinear fields. The dielectric rods are inserted in a boric anhydride $\mathrm{B}_{2} \mathrm{O}_{3}$ doped silica substrate material. Figure 1 describes the cross-sectional view of the designed PCF with different doping.

These visualizations serve as a useful tool for obtaining quantitative data on mode frequency response. Computing the eigenvector (field) corresponding to each eigenvalue $\left(\mathrm{n}_{\text {eff }}=\beta / K_{0}\right)$ yields descriptive data of dispersion, effective area, and normalized frequency. Figure 2 shows the neff of hexagonal PCF for varying concentrations of $\mathrm{B}_{2} \mathrm{O}_{3}$ (e.g., $3.0 \%, .5 \%$, nd $13.5 \%$ ) nd nonlinearities. Figures 3,4 , and 5 correspond to emerging trends in dispersion, effective area, and normalized frequency. Table I shows the Sellmeier coefficients of $\mathrm{B}_{2} \mathrm{O}_{3}$ doped materials. Basic parameters of the PCF include core, cladding, and air cladding diameter which are adjusted at 10, $40 \& 41 \mu \mathrm{m}$. For mode analysis, a substantially higher index material (SF57- dielectric-rods) with a relatively high filling factor in cladding has been chosen to design dispersion-compensating PCF. The diameter of SF57 dielectric rods was increased to $1.44 \mu \mathrm{m}$ while the rod filling factor was retained at 0.8 . The pulse width of the source signal has also been set at $4.5 \mu \mathrm{m}$ for the assessment of effective refractive index and field. We focused on dispersion, effective area, and normalized frequency for the same design but with varied source intensities and molar concentrations of $\mathrm{B}_{2} \mathrm{O}_{3}$ in the substrate of the fiber. The study was established across a visible spectrum of 0.5 to 0.8 meters. Sellmeier's expression given in [17] was used to measure the refractive index of SF57 glass as well as substrate materials. The values of pitch $=3.6 \mu \mathrm{m}$ and $d=1.44 \mu \mathrm{m}$ have been chosen because they provide a better trade-off between flattened dispersion and PCF numerical aperture range. The content of $\mathrm{B}_{2} \mathrm{O}_{3}$ in the core and background cladding of the designed PCF has been altered, but the 
geometry and basic properties of the fiber have remained unchanged. The refractive indexes of these materials have one of the great characteristics of wavelength addiction, with different refractive indexes for different content of doped material in core and cladding. In the cladding zone, the use of lead silicate SF57 rods in place of air-rods results in a high neff of cladding. The PCF integrated into this approach follows the PBG guiding mechanism, and the wave is captured internally within the core region. However, for varying intensity $(I)$ when the refractive index of the core becomes equal or smaller than the refractive index of cladding, no more the condition of PBG wave guiding fits. The effective refractive index tends toward a greater value attributable to the severe nonlinearity, resulting in a substantial change in dispersion and PCF effective area. From the $\mathrm{n}_{\text {eff }}$ versus $\lambda$ curve, demonstrated in Figure 2 , it is clear that for different mole $\%$ of doped material, the slope of $n_{\text {eff }}$ is typically distinct.

For waveguiding in a corridor with constrained transverse extension, the effective refractive index $n_{\text {eff }}$ seems to have the same interpretation as for plane wave inhomogeneous transparent media. Effective refractive index measures the phase delay per unit length in a waveguide in comparison to phase delay in a vacuum. In atypical PCF, it depends not only on the phase constant modulated by wavenumber but also on the spatial distribution of dielectric rods in PCF and their fill factor. As the filling factor of SF57 increases, the contribution of dielectric rods material in matrix element grows, culminating in a high value of $\mathrm{n}_{\text {eff }}$ at the operating frequency. Material dispersion is caused by the material's refractive index's dependence on wavelength. Waveguide dispersion in fiber occurs due to disparity in refractive index between core and cladding. When the effective refractive index of the cladding is bigger than it is to the core, core photons propagate quicker. We can conclude from Figure 3 that $\mathrm{B}_{2} \mathrm{O}_{3}$ doped silica PCFs have a significant impact on dispersion gradient and may be tailored to around zero-dispersion by selecting the appropriate mole percentage of doped material. The small core diameter, and high filling factor of SF57 glass rods, is flattening dispersion. In comparison to low intensity, the computational findings imply that dispersion for the intermediate fraction of doped material flattens down towards zero at high intensity. Figure 3 shows a substantial normal dispersion in the midpoint of the selected range, as represented by the upward curving of the dispersion curve. These findings indicate that PBG-PCFs with proper boron doping get the propensity to exhibit substantial atypical dispersion.

When a beam travels across an optically active region, its pulse width fluctuates in the direction perpendicular. In the analysis, the input pulse width of the Gaussian beam has been considered, $0.45 \mu \mathrm{m}$ and occurring changes have been measured in Figure 4 for different intensity and molar content of doped material. For differing doped material levels and intensities, it exhibits linear response at a chosen range of wavelength. The effective area grows as the intensity increases due to the nonlinear index variation of core and cladding. $\mathrm{B}_{2} \mathrm{O}_{3}$ doping lowers field trapping in the cladding region in comparison to ordinary silica PCF. These results depict the field distribution in hexagonal PCF's cross-sectional structure. Longer wavelengths enable the field to transpose into the core, leading to the reduced effective area in the PCF core. 
The normalized frequency (V-parameter) is the last optical parameter, we determine from $\mathrm{n}_{\text {eff }}$ data collected in Fig. 2. The expression for normalized frequency for the PCF governing nonlinear waves is influenced by both intensity-dependent core and cladding refractive index. The curve in Figure 5 depicts the effect of change of molar contents of doped material and intensities on V-parameter. It is straightforward from these figures that by carefully selecting the concentration of doped material, it is possible to keep normalized frequency, $\mathrm{V}$ below a cut-off value for the entire range of wavelength mentioned. The PCF meets the criteria of multimode operation if the value is greater than this cut-off value.

\section{Conclusion}

In this article, we modeled the hexagonal PCF using SF57 for a different molar concentration of $\mathrm{B}_{2} \mathrm{O}_{3}$ such as $3.0 \%, 3.5 \%, 13.5 \%$, and different nonlinearities. The investigation of dispersion, effective area and normalized frequency for this class of PCF are widely researched in the presence of nonlinear sources. We measured the effective area as a function of wavelength for the suggested structure at varying intensities and doped material contents. A PCF of this type could provide a potential substrate for discrete mode laser diodes generating multi-mode at wavelengths of $=0.65 \mathrm{~m}$. The numerical results show that the effective area of PCF with $\mathrm{B}_{2} \mathrm{O}_{3}$ doped silicate varies linearly with wavelength, but it has a higher value for the same intense field when the mole percentage of $\mathrm{B}_{2} \mathrm{O}_{3}$ is increased. For low doping of $\mathrm{B}_{2} \mathrm{O}_{3}$, it has boosted and significantly enhanced beam confinement with nearly flattened dispersion for distal non-linearity.

\section{Declarations}

\section{Acknowledgement:}

This work was partially supported by the AICTE Collaborative Research Scheme, India through application ID 1-5730324531.

\section{References}

1. Luo, W., et al.: "Long-Range Surface Plasmon Resonance Sensor based on side-polished D-shaped hexagonal structure photonic crystal fiber with the buffer layer of magnesium fluoride." Journal of Physics D: Applied Physics (2021)

2. Dixit, A., et al.: Refractive index sensor based on evanescent field effects in hollow core PCF for detection of analytes over extended $\mathrm{E}+\mathrm{S}+\mathrm{C}+\mathrm{L}+\mathrm{U}$ communication bands. Opt. Laser Technol. 121, 105779 (2020)

3. Rajasekar, R., Robinson, S.. "Nano-pressure and temperature sensor based on hexagonal photonic crystal ring resonator." Plasmonics 14.1: (2019): 3-15 
4. Lou, J., Cheng, T., Li, S.: Ultra-short polarization beam splitter with square lattice and gold film based on dual-core photonic crystal fiber. Optik 179, 128-134 (2019)

5. Vyas, A.K.: Multiple rings based photonic crystal fiber for terahertz application. Optik 231, 166424 (2021)

6. Liu, Q., Xin, L., Wu, Z.: Refractive index sensor of a photonic crystal fiber Sagnac interferometer based on variable polarization states. Appl. Phys. Express 12(6), 062009 (2019)

7. Lakshminarayana, G., Meza-Rocha, A.N., Soriano-Romero, O., Huerta, E.F., Caldiño, U., Lira, A., Lee, D.E.: Jonghun Yoon, and Taejoon Park. "Survey of optical and fluorescence traits of Tm3+-doped alkali/mixed alkali oxides constituting B2O3-BaO-ZnO-LiF glasses for $0.45 \mu \mathrm{m}$ laser and $1.46 \mu \mathrm{m}$ fiber amplifier." Results in Physics (2021): 104343

8. Limpert, J., et al.: High-power air-clad large-mode-area photonic crystal fiber laser. Opt. Express 11(7), 818-823 (2003)

9. Kumar, C., Kumar, G.: S+ C double-band flattened gain hybrid optical amplifier [RAMAN+ thuliumdoped photonic crystal fiber amplifier (TD-PCFA)] for super-dense wavelength division multiplexing system. J. Opt. 49(2), 178-180 (2020)

10. Liu, X., et al.: Stable and uniform dual-wavelength erbium-doped fiber laser based on fiber Bragg gratings and photonic crystal fiber. Opt. Express 13(1), 142-147 (2005)

11. Limpert, J., et al.: Low-nonlinearity single-transverse-mode ytterbium-doped photonic crystal fiber amplifier. Opt. Express 12(7), 1313-1319 (2004)

12. Beugin, V., et al.: "Efficient Bragg gratings in phosphosilicate and germanosilicate photonic crystal fiber." Applied optics 45.32 (2006): 8186-8193

13. Dixit, A., Tiwari, S., Praveen Chandra, P.: Optical properties of 3rd order Kerr hexagonal nonlinear photonic crystal fiber containing metal. Int. J. Mod. Phys. B 31, 8 (2017). "." ( : 1750047.

14. Olszewski, J., et al.: "Photonic crystal fibre with $\mathrm{GeO} 2$ and $\mathrm{B} 2 \mathrm{O} 3$ doped inclusions for compensating chromatic dispersion." Finds and Results from the Swedish Cyprus Expedition. A Gender Perspective at the Medelhavsmuseet (2008)

15. Zhang, Y., et al.: Porous photonic-crystal fiber with near-zero ultra-flattened dispersion and high birefringence for polarization-maintaining terahertz transmission. Optik 207, 163817 (2020)

16. Zhang, Y., Jing, X., Qiao, D.: Lu Xue. "Rectangular porous-core photonic-crystal fiber with ultra-low flattened dispersion and high birefringence for terahertz transmission." Front. Phys. 8 (2020): 370

17. Khoobjou, E., Khalesi, H., Ghods, V.: An optimal design for decreasing dispersion in photonic crystal fiber. Journal of Electrical Engineering \& Technology 15(6), 2691-2698 (2020)

18. Li, J., et al.: Broadband supercontinuum generation based on filled structural photonic crystal fibers with low incident optical power. Opt. Quant. Electron. 52, 1-11 (2020) 10 )

19. Reeves, W.H., Knight, J.C., St, P., Russell, J.: and P. J. Roberts. "Demonstration of ultra-flattened dispersion in photonic crystal fibers. Optics express 10, 609-613 (2002).", no. 14 ) 
20. Mortensen, N.A., et al.: Improved large-mode-area endlessly single-mode photonic crystal fibers. Opt. Lett. 28(6), 393-395 (2003)

21. Matsui, T., et al.: Dispersion-flattened photonic crystal fiber with large effective area and low confinement loss. J. Lightwave Technol. 23(12), 4178-4183 (2005)

22. Mortensen, N.A.: "Effective area of photonic crystal fibers.". Opt. Express 10, 341-348 (2002) 7 )

23. Knight, J.C., et al.: Properties of photonic crystal fiber and the effective index model. JOSA A 15(3), 748-752 (1998)

24. Knight, J.C., et al.: Properties of photonic crystal fiber and the effective index model. JOSA A 15(3), 748-752 (1998)

25. Himei, Y., et al.: Thermo-optic properties of B2O3 doped Li2O-Al2O3-SiO2 glass-ceramics. J. Noncryst. Solids 354(27), 3113-3119 (2008)

26. Napiorkowski, M.: and Waclaw Urbanczyk. "Effect of bending on surface plasmon resonance spectrum in microstructured optical fibers. Optics express 21, 22762-22772 (2013) 19)

27. Tiwari, S., Dixit, A., Praveen, C.: Pandey. "Effect of Dispersive Materials on the Dispersion and Normalized Frequency Characteristics of a Square Photonic Crystal Fiber. Journal of Nanoelectronics and Optoelectronics 11(6), 709-714 (2016)

28. Bruner, A., et al.: Temperature-dependent Sellmeier equation for the refractive index of stoichiometric lithium tantalate. Opt. Lett. 28(3), 194-196 (2003)

29. Yu, J.W., Oh, K.: New in-line fiber band pass filters using high silica dispersive optical fibers. Optics communications 204, 1-6 (2002). "." ( : 111-118.

30. Xu, Q., Runcai Miao, and Zhang, Y.: Highly nonlinear low-dispersion photonic crystal fiber with high birefringence for four-wave mixing. Opt. Mater. 35(2), 217-221 (2012)

31. Hwang, I.-K., Lee, Y.-J.: and Yong-Hee Lee. "Birefringence induced by irregular structure in photonic crystal fiber." Optics Express 11.22 (2003): 2799-2806

32. Tiwari, S., Dixit, A., Praveen, C.: Pandey. "Effect of Dispersive Materials on the Dispersion and Normalized Frequency Characteristics of a Square Photonic Crystal Fiber. Journal of Nanoelectronics and Optoelectronics 11, 709-714 (2016) 6 )

33. Mortensen, N., Asger, et al.: "Numerical aperture of single-mode photonic crystal fibers.". IEEE Photonics Technol. Lett. 14, 1094-1096 (2002) 8 )

34. Vyas, A.K.: Elliptical air holes based photonic crystal fiber for narrow band gap and peak power at 1.55 micrometre wavelength. Optik 184, 28-34 (2019)

\section{Figures}




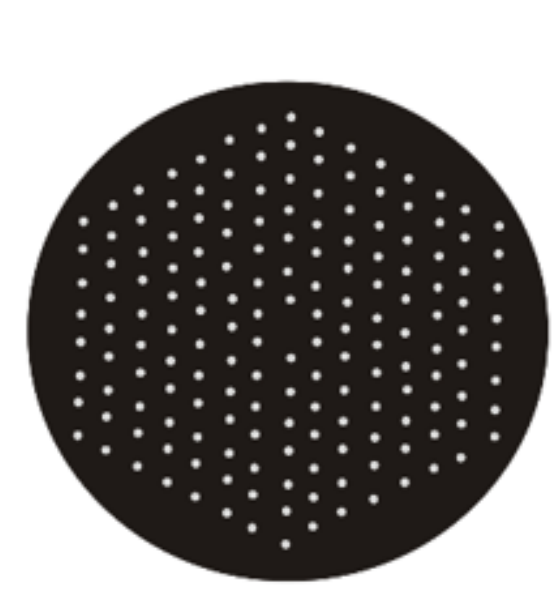

SF 57 with $3.0 \%$

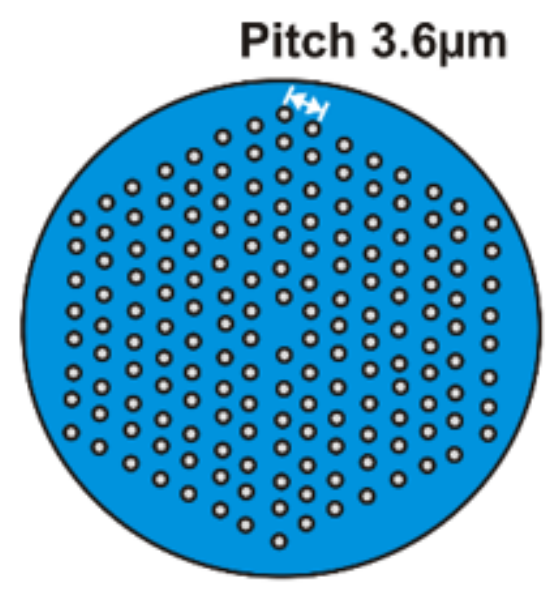

SF 57 with $13.0 . \%$

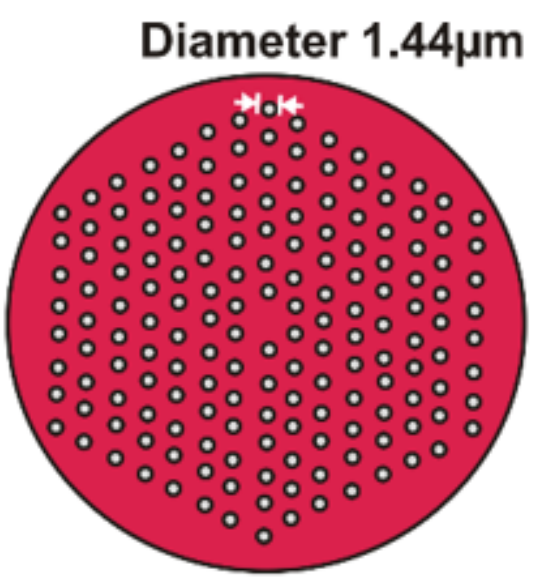

SF 57 with $3.5 \%$

Figure 1

Cross-sectional view of the proposed model with different doping concertation. 

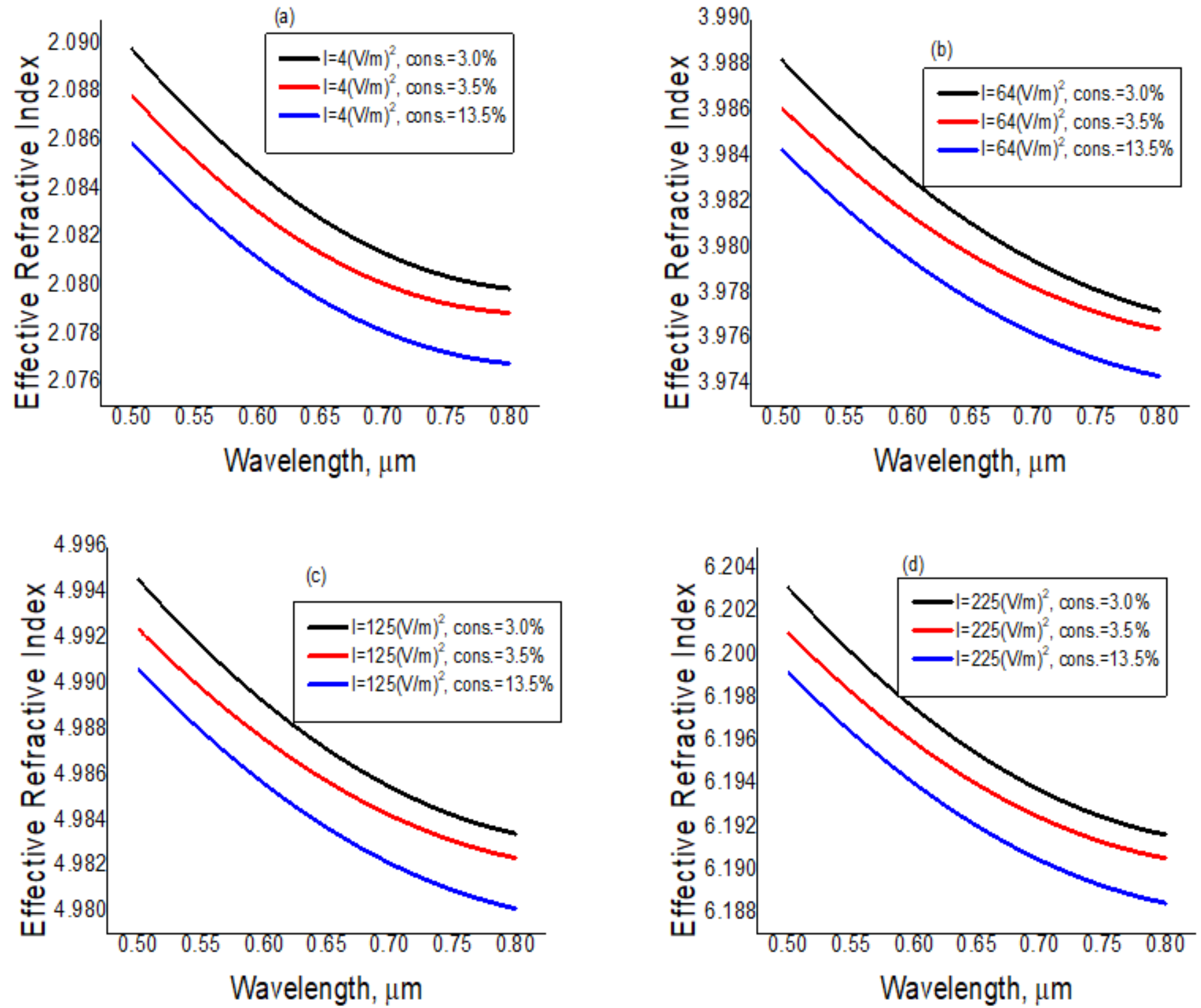

Figure 2

Effective refractive index versus wavelength curves for different intensities and doping of boric anhydride in silica. 

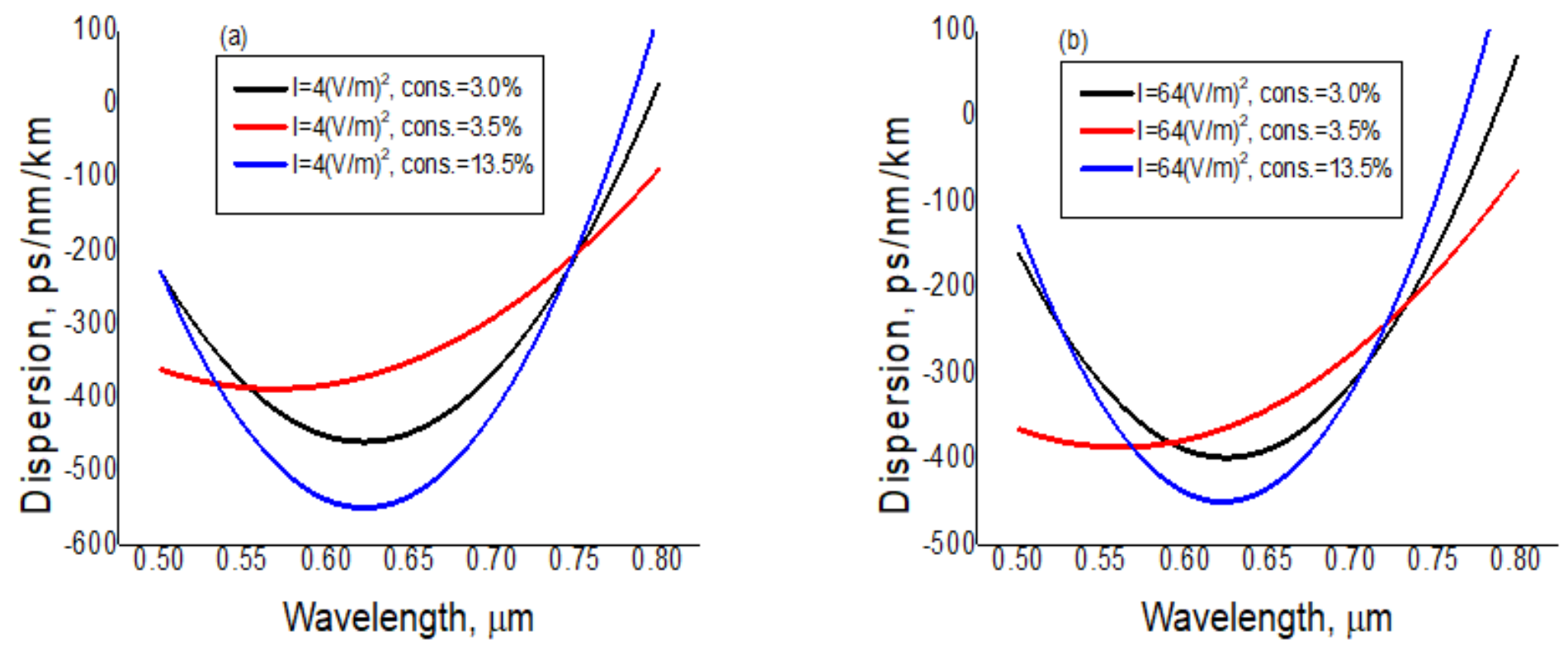

Figure 3

Dispersion versus wavelength curves for different intensities and doping of boric anhydride in silica.
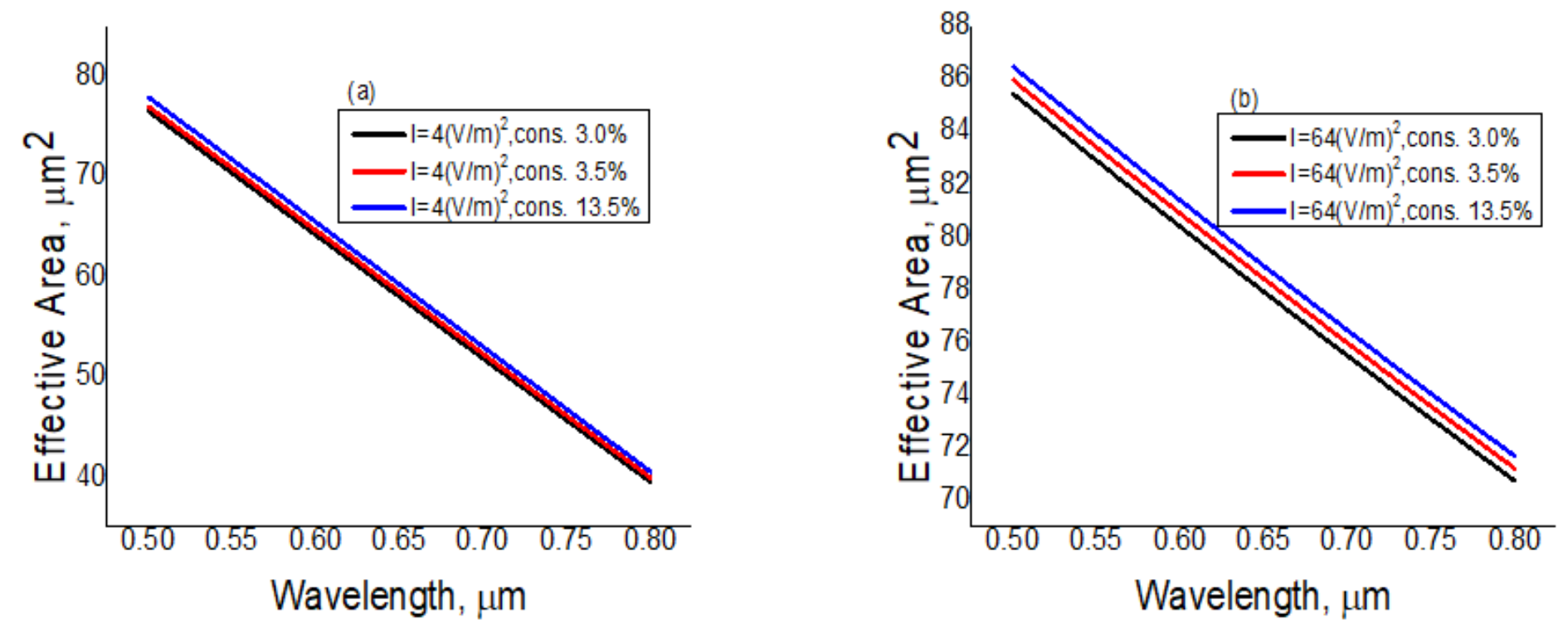

Figure 4

Effective area versus wavelength curves for different intensities and doping of boric anhydride in silica. 

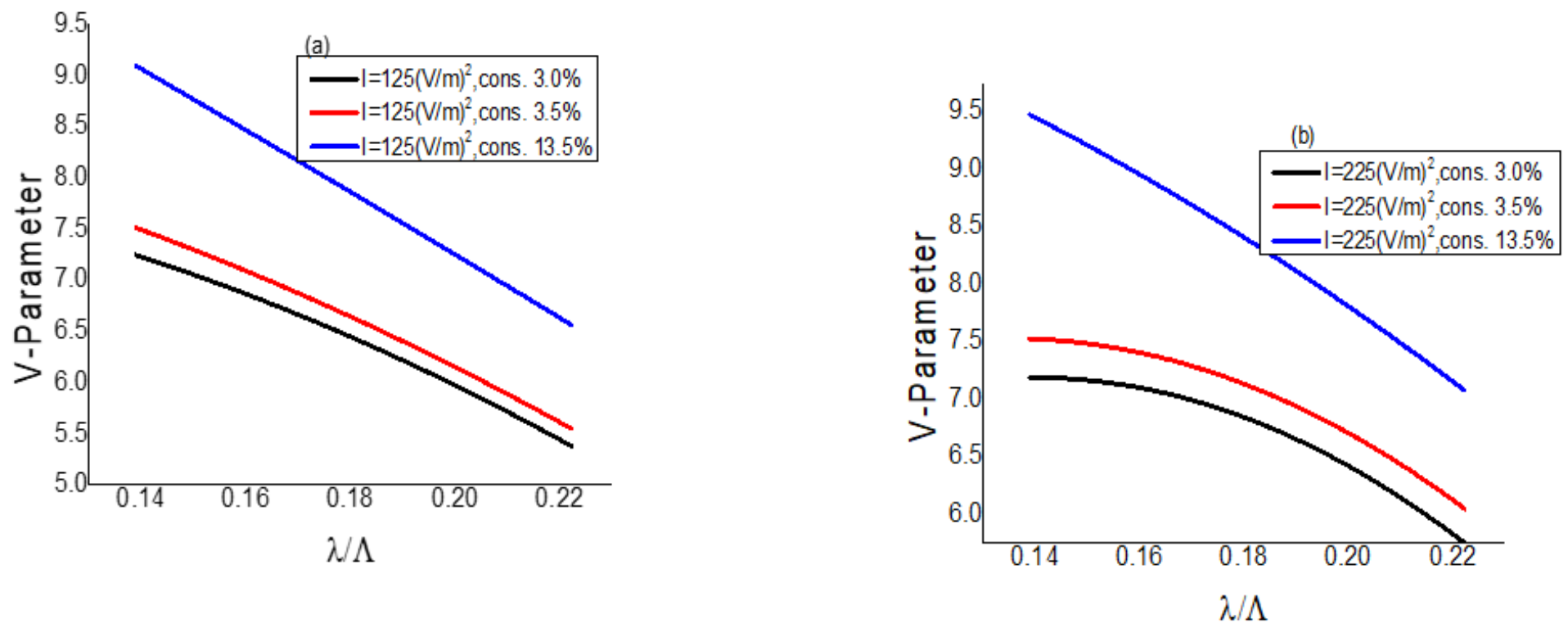

Figure 5

Normalized frequency versus normalized wavelength curves for different intensities and doping of boric anhydride in silica. 